\title{
Proposal of Abnormal Sound Detection System using OCSVM
}

\author{
Hikaru Ito ${ }^{\text {a }}$ Fumiaki Ehara ${ }^{\text {a, }}$, Yoshihiko Muto ${ }^{\text {a }}$

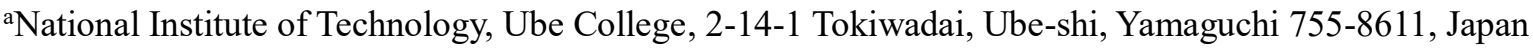 \\ *Corresponding Author: ehara@ube-k.ac.jp
}

\begin{abstract}
In order for a machine to operate safely, it is one of the very important factors to quickly and accurately detect an abnormality. However, it is difficult to detect accurately and always monitor the machine by human decision. Daily inspections of machines are generally performed by monitoring images, vibrations, temperature and pressure. However, it is commonly thought that inspections using sound is difficult because sound is different how to hear depending on the person. In addition, machine learning came to be used easily on these days.

In this study, we examined whether machine abnormalities can be detected using machine learning. To prepare for the study, the abnormal machine sounds were needed. However, it is difficult to collect the abnormal machine sound. In this study, to generate the abnormal sound easily, we build a machine operation sound generator. Some abnormal sounds were generated by applying a load to this device. We extracted features called Mel-Frequency Cepstrum Coefficient(MFCC) from machine operation sounds. MFCC is one of the features and is popular as a method of sound recognition. Subsequently, abnormal sounds were detected by using One Class Support Vector Machine (OCSVM). OCSVM is a method to regard normal data as one class and detect other than normal data. The one of the merits of OCSVM can detect abnormal data by using results of learning only normal data.

We conducted experiments to confirm the effectiveness of the proposed system. We assumed that the sounds of first day were normal sounds and the other sounds assumed abnormal sounds. As a result, the abnormality rate on the fourth day was $78.94 \%$ and we conclude that abnormal sounds could be detected from machine operation sounds.
\end{abstract}

Keywords: Abnormal Sound Detection, Machine Operation Sound Generator, MFCC, One Class SVM

\section{Introduction}

In order to operate the machine safely, it is very important to detect an abnormality of the machine quickly and accurately. However, human abnormal detection is not accurate and it is difficult to always monitor the machine. Therefore, we thought that it could be possible to detect machine abnormalities using machine learning that is currently attracting attention. In this study, machine operation sounds measured in advance were converted to features called Mel-Frequency Cepstrum Coefficient (MFCC). We examined the abnormal sound detection by using the MFCC and One-Class Support Vector Machine (OCSVM) which is one of the machine learning and is popular as an abnormality detection method.

\section{Machine Operation Sound Measurement}

The rough flow of this study is shown in Fig.1. We measured the machine operation sounds using sound generator. The section 2 described how to measure the machine operation sounds.

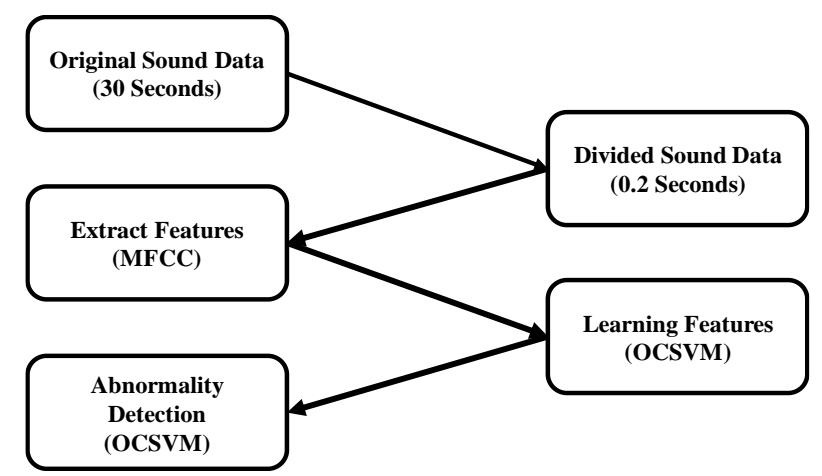

Fig. 1. Flow of System 


\subsection{Machine Operation Sound Generator}

The machine operation sound generator is a device in which power is transmitted to the drive shaft of Fuji lowpressure three-layer premium efficiency motor MLK1085M through a flexible coupling, and two bearings are installed on the shaft. Some abnormal sounds can be generated by freely changing the power supply frequency of the motor using an inverter and controlling the number of rotations or applying a load to the drive shaft. Connect the signal from the contact microphone attached to the bearing to the analog input terminal of the $\mathrm{I} / \mathrm{O}$ device and measure two simultaneously.

Fig. 2 is a schematic diagram of the machine operation noise generator. The length of device is about two meters and the width is about one meter. Fig. 3 is a schematic view of how the drive shaft is applied a load. As shown in Fig.3, the load is applied to generate an abnormal sound by shifting the drive shaft. The machine operation sounds were measured during applying the load. By applying the load, the machine operation sounds were changed gradually. We assumed that

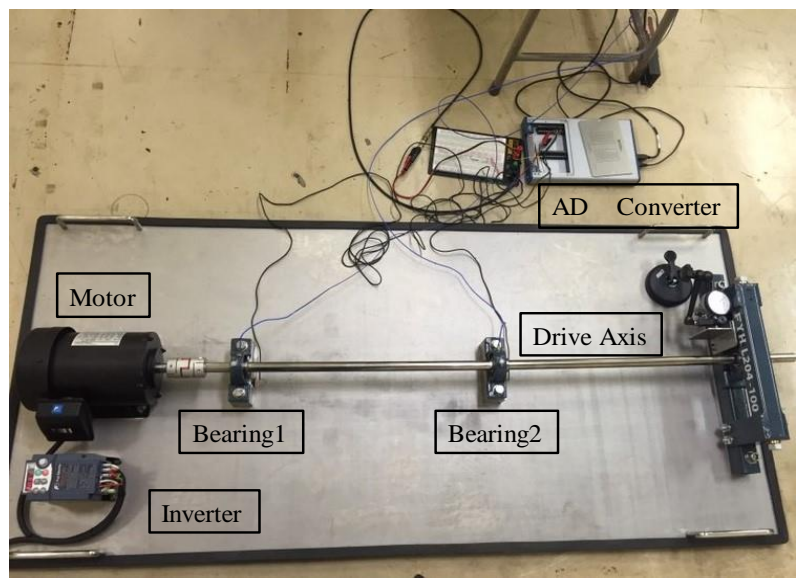

Fig. 2. Machine Operation Sound Generator

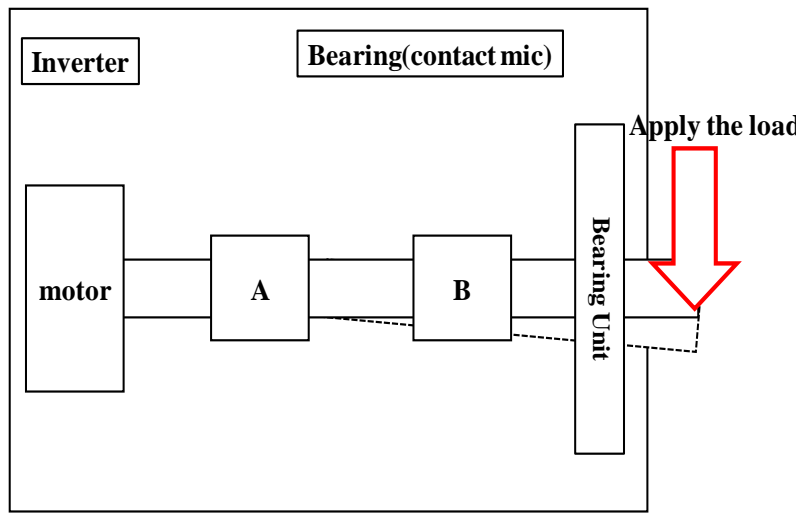

Fig. 3. Schematic of Placing the Drive Shaft the first few sounds were normal and the others were abnormal.

\subsection{Machine Operation Sound}

Machine operation sounds were measured using MATLAB. The sampling frequency is $50[\mathrm{kHz}]$, and the measurement time is 30 seconds. Machine operation sounds are applied by shifting the drive shaft by $10 \mathrm{~mm}$ and measuring data for 30 seconds every 20 minutes. The sounds were measured during applying the load in four days. The total measurement time is 9870 seconds. Measured machine operation sounds time of each day are shown in Table1.

In this study, we assumed that the sounds of first day were normal and the others were abnormal. We divided these 30 seconds of sound data into 0.2 seconds and made into 150 sound data. The 0.2 seconds machine operation sound sample is shown in Fig4. Amplitude spectrums of normal sound and abnormal sounds are shown in Fig5.

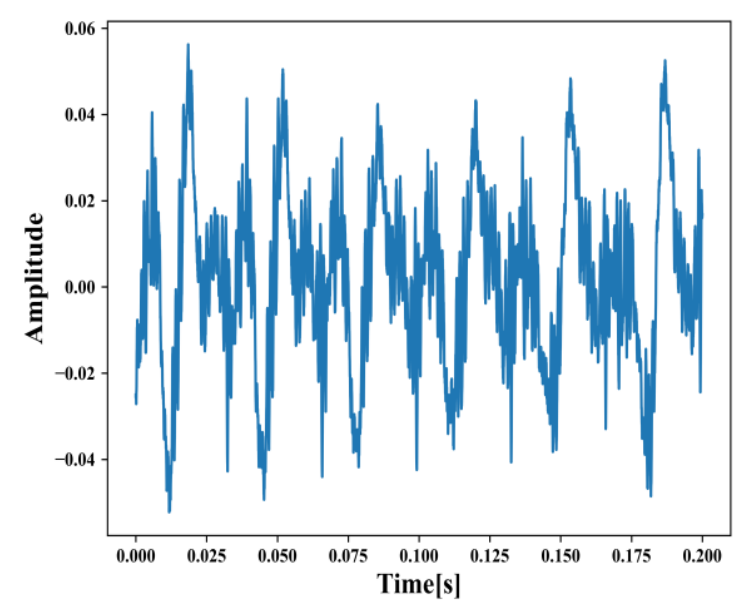

Fig. 4. 0.2 Seconds Machine Operation Sound

Table 1. Measurements Time.

\begin{tabular}{c|cccc|c}
\hline & Day1 & Day2 & Day3 & Day4 & Total \\
\hline $\begin{array}{c}\text { Number of } \\
\text { Measurements }\end{array}$ & 45 & 54 & 147 & 93 & 339 \\
Time[s] & 1350 & 1620 & 4410 & 2490 & 10170 \\
\hline
\end{tabular}




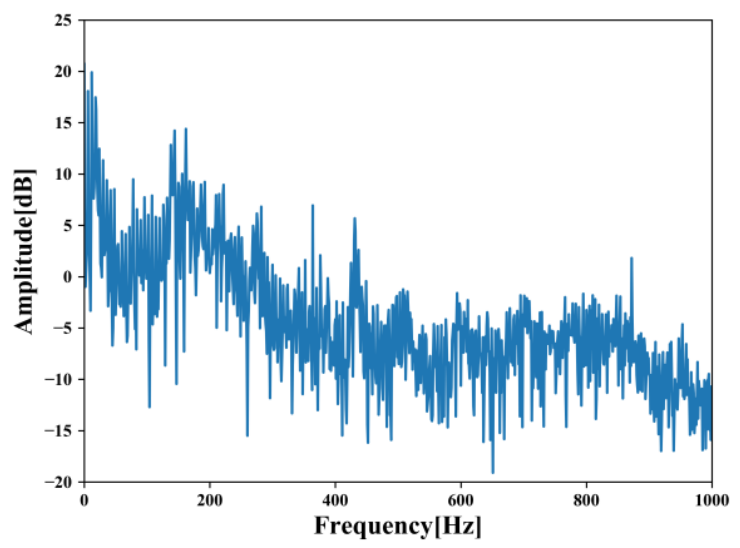

(a) Normal Sound.

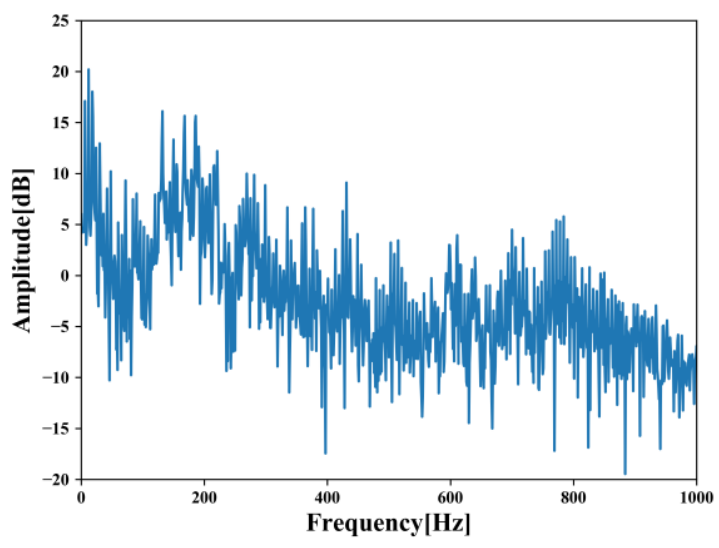

(b) Abnormal Sound.

Fig. 5. Amplitude Spectrum.

\section{Abnormal Sound Detection Program}

In this study, an abnormal sound detection program was created using python. From the machine operation sound, features called MFCC was extracted, and then abnormality detection was performed by One-Class SVM. The section 3 described how to extract the features and detect the abnormality of machine operation sound.

\subsection{Mel Frequency Cepstram Coefficient (MFCC)}

Mel-Frequency Cepstram Coefficient (MFCC) is features that take into consideration the characteristics of speech perception in a cepstrum representing human vocal tract characteristics. Our goal is to detect the abnormal sound from the machine operation sounds. There are many types of machines. It is necessary to use features that can be applied to all machines. We selected MFCC and validated whether it could detect abnormal sound because MFCC is often used in

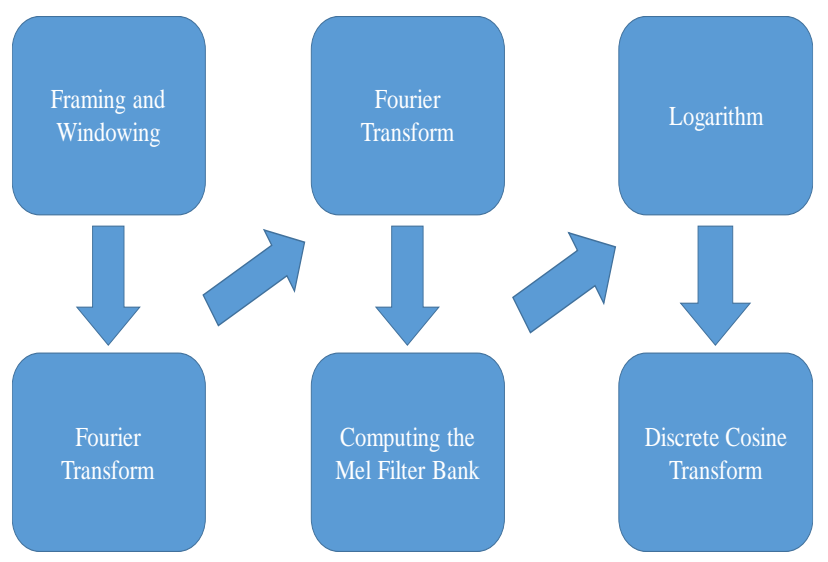

Fig. 6. Flow of MFCC Extraction

the field of speech recognition.

The flow of MFCC extraction is shown in Fig.6. The 19 point $\times 20$ frame MFCC (380) was extracted from 0.2 seconds of sound data. Applying these processes to all sound data, a total of $380 * 49350(18,753,000)$ MFCC were extracted. Abnormal sound detection is performed using these extracted features.

\subsection{One Class Support Vector Machine}

OCSVM is unsupervised learning that detects outliers ${ }^{(1)}$. Unsupervised learning is learning in which no correct answer label is given to training data. OCSVM is a learning method that normalizes the initially given data and detects values outside of it.

Fig.7 shows a conceptual diagram of $\mathrm{OCSVM}^{(2)}$. Fig.7(a) shows the space of the original input data. Normal data (red circles in the figure) exist in some group, but abnormal data (blue circles in the figure) exist scattered. By passing a certain mapping function, as shown in Fig.7(b), the normal data are converted to one place, and the abnormal data are converted to a position away from normal data. In the OCSVM, the optimal center coordinates and radius of the hypersphere are calculated in the space after this mapping.

The advantage of OCSVM is that the data used for training is only normal data. It is difficult to collect abnormal machine operation sounds. Therefore, we chose OCSVM to detect abnormal sound. In this study, features of 1350 seconds on the first day was used as training data (normal sound), and features of 8820 seconds for the remaining 3 days was used as test data. Since these features were extracted every 0.2 seconds, training and testing are performed every 0.2 seconds. 
Table 1. Execution Result.

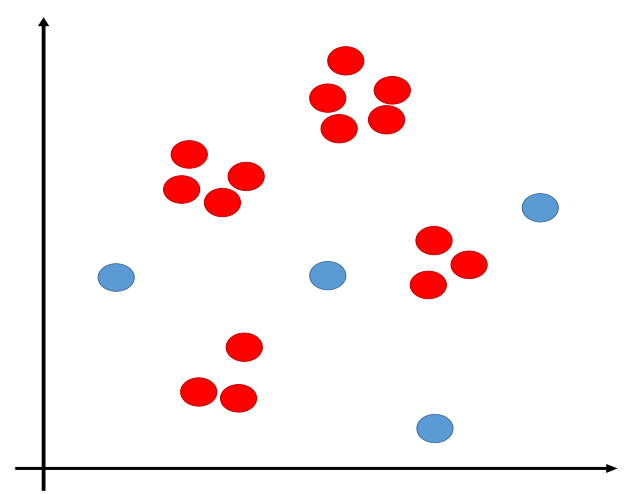

(b) Space of the Original Input Data.

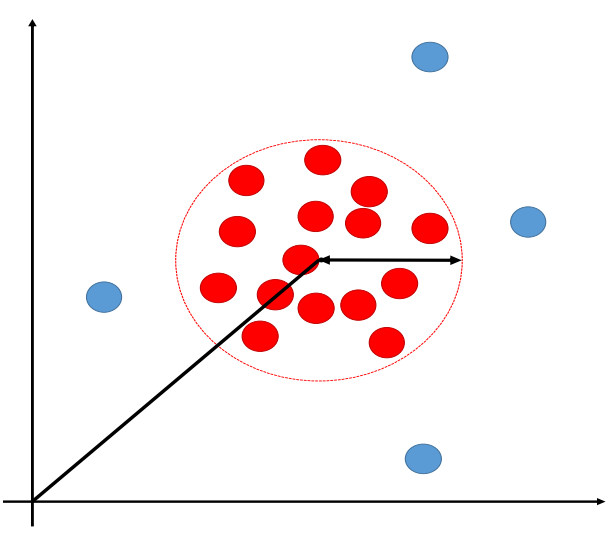

(b) Feature Space.

Fig. 5. Conceptual Diagram of OCSVM.

\section{Execution Result}

The execution results are shown in Table 2. The numerical values in Table 2 are the number of features every 0.2 seconds, and are the number of normality and the number of abnormality judged by OCSVM. The percentage of abnormality for each day are shown in the Fig.1. The percentage of abnormality of the measured machine operation sounds were $54.00 \%$ on the second day, $54.31 \%$ on the third day, and $78.97 \%$ on the fourth day. As shown in Figure 6 , the percentage of abnormality tends to increase over time.

\section{Conclusions}

In this study, we examined whether it is possible to perform anomaly detection using OCSVM. The machine operation noise was measured, the feature quantity was extracted, and then the abnormal sound was successfully detected by machine learning. From these results, it was

\begin{tabular}{|c|c|c|c|}
\hline & Day2 & Day3 & Day4 \\
\hline Total & 8100 & 22050 & 12450 \\
\hline Normality & 3726 & 10074 & 2618 \\
\hline Abnormality & 4374 & 11976 & 9832 \\
\hline \multicolumn{4}{|l|}{90.00} \\
\hline \\
\hline \multicolumn{4}{|l|}{40.00} \\
\hline \\
\hline \multicolumn{4}{|l|}{10.00} \\
\hline \multirow{3}{*}{0.00} & & & \\
\hline & & & Day4 \\
\hline & Meas & & \\
\hline
\end{tabular}

Fig. 6. Execution Result Trend.

found that machine abnormalities can be detected by using a system that measures machine operation sounds, extracts features, and machine learning.

However, since this experiment was performed using a device for generating machine operation sounds, it is questionable whether it can be adapted to the actual operation sounds of various machines. Therefore, it is necessary to collect the operating sounds of the machine actually used and examine whether this system can be adapted. Also, there are many parts that can be improved, such as the selection of features and the number of sound data divisions. I would like to optimize these and improve the accuracy in the future.

\section{References}

(1) Tsuyoshi Ide, and Masashi Sugiyama: "Anormaly Detection and Change Detection", pp.65-77,2015

(2) Ken-ichi Fukui: "Machine learning with python and examples. Identification, prediction, anomaly detection", pp.87-120, 2018 